\title{
A GEANT-based study of atmospheric neutrino oscillation parameters at INO
}

\author{
Abhijit Samanta, ${ }^{1}$ Sudeb Bhattacharya, ${ }^{1}$ Ambar Ghosal, ${ }^{1}$ Kamales Kar, ${ }^{1}$ Debasish Majumdar, ${ }^{1}$ and Amitava Raychaudhuri ${ }^{2,3}$ \\ ${ }^{1}$ Saha Institute of Nuclear Physics, 1/AF, Bidhannagar, Kolkata 700 064, India \\ ${ }^{2}$ Harish-Chandra Research Institute, Chhatnag Road, Jhusi, Allahabad 211 019, India \\ ${ }^{3}$ Department of Physics, University of Calcutta, 92 Acharya Prafulla Chandra Road, Kolkata 700 009, India
}

(Dated: December 24, 2018)

\begin{abstract}
We have studied the dependence of the allowed space of the atmospheric neutrino oscillation parameters on the time of exposure for a magnetized Iron CALorimeter (ICAL) detector at the India-based Neutrino Observatory (INO). We have performed a Monte Carlo simulation for a $50 \mathrm{kTon}$ ICAL detector generating events by the neutrino generator NUANCE and simulating the detector response by GEANT. A chi-square analysis for the ratio of the up-going and down-going neutrinos as a function of $L / E$ is performed and the allowed regions at $90 \%$ and $99 \% \mathrm{CL}$ are displayed. These results are found to be better than the current experimental results of MINOS and Super-K. The possibilities of further improvement have also been discussed.

PACS numbers: $14.60 . \mathrm{Pq}, 96.40 . \mathrm{Tv}$
\end{abstract}

\section{INTRODUCTION}

The evidence of neutrino masses and their mixing [1, 2] has brought neutrino physics into centre stage of particle physics. The neutrino mass eigenvalues and the Pontecorvo, Maki, Nakagawa, Sakata (PMNS) mixing matrix [3, 4] connecting the mass to the flavor basis provides a natural framework for handling three active neutrinos.

The present information on the neutrino mass-squared differences and mixing angles are the following: From atmospheric neutrino detection one gets the best-fit values with $3 \sigma$ error $\left|\Delta m_{32}^{2}\right| \simeq 2.5_{-0.6}^{+0.7} \times 10^{-3} \mathrm{eV}^{2}, \sin ^{2} \theta_{23} \simeq 0.5_{-0.11}^{+0.18}$ while solar neutrinos tell us $\Delta m_{21}^{2} \simeq 7.9 \times 10^{-5} \mathrm{eV}^{2}, \sin ^{2} \theta_{12} \simeq 0.30$ [5]. Here we define $\Delta m_{i j}^{2}=m_{i}^{2}-m_{j}^{2}$.

At the moment, the sign of $\Delta m_{32}^{2}$ is not known. The positive/negative value of this quantity denotes the direct/inverted mass ordering. The two large mixing angles and the mass squared differences may permit measurement of $\mathrm{CP}$-violation in the lepton sector, if the third mixing angle, $\theta_{13}$, and the CP phase, $\delta$, are not too small. The current bound on the former is $\sin ^{2} \theta_{13}<0.05(3 \sigma)[6,7]$ while $\delta$ is unconstrained.

Thus the determination of mass hierarchy and the measurement of oscillation parameters with high precision are of utmost importance. Also of importance is observing a full oscillation cycle to convincingly establish that it is truly neutrino oscillation which is at play. Most experiments observe the depletion part but not the regeneration part of the cycle. A reanalysis of old Super-Kamiokande (Super-K) data claimed to observe this [8]. However, a reconfirmation of this with better statistics is much awaited. The mixing angle and the mass squared difference for the atmospheric sector should also be measured more accurately. The sensitivity of the measure- ment of a particular parameter depends crucially on the ranges of neutrino energy and path length traversed from the source to the detector. These ranges can be set in case of neutrino beams from artificial sources like nuclear reactors (energy $\sim$ $\mathrm{MeV}$ ) and accelerators (energy $\sim \mathrm{GeV}$ ). Neutrinos with energy $\sim \mathrm{MeV}(\mathrm{GeV})$ can also be obtained from natural sources like the sun (the atmosphere). Unlike typical accelerator or reactor neutrinos, the spectrum of atmospheric neutrinos covers many decades of energy ( $E \sim 100 \mathrm{MeV}$ - few hundred $\mathrm{GeV})$ with comparable interaction rate and baseline $(L \sim 10$ $\mathrm{km}-12800 \mathrm{~km})$. Since the oscillation probability depends mainly on $L / E$ which varies in a wide range for atmospheric neutrinos, the measurement of the appearance/disappearance probability as a function of $L / E$ can explore its variation over this entire range. This advantage is partly offset, however, by the difficulty that the flux is less known compared to that from man-made sources.

Currently around the world, there are many ongoing and planned experiments: MINOS [9, 10], T2K [11], ICARUS [12, 13], NOvA [14, 15], Double Chooz [16, 17], UNO [18], Super-K III [19], Hyper-K [20, 21], OPERA [22, 23, 24] etc. Out of these only MINOS employs a magnetic field and has a good charge identification capability. It is to be noted that all these experiments are planned in the northern hemisphere of the earth.

The proposed India-based Neutrino Observatory (INO) [25] at a site close to the equator plans to use a large magnetized Iron CALorimeter (ICAL) detector. The proposal is for an underground facility with more than $1 \mathrm{~km}$ overburden. Since the detector has a high charge identification capability (>90\% after selection of events as described in section $\mathrm{V}$ and $70 \%$ before doing the selection) [25], it has a good chance 
of determining the neutrino mass ordering [26, 27, 28, 29] and also of studying the deviation from maximality for $\theta_{23}$ [30, 31].

In this work we first demonstrate through a GEANT-based simulation of atmospheric neutrinos that ICAL indeed is capable of observing the full oscillation cycle. We have used a two flavor oscillation formalism and studied the precision that can be achieved for $\left|\Delta m^{2}\right|$ and $\sin ^{2} 2 \theta$ at INO with atmospheric neutrinos. Though a more realistic approach would be the use of three flavor analysis, the smallness of the mixing angle $\theta_{13}$ ensures that the two flavor approximation mimics the real situation reasonably well. The precision depends on the exposure in terms of kTon-yr, reconstruction method, and the selection of the events in the analysis. The paper is organized as follows: A brief summary of the neutrino oscillation formalism is given in Section II. The ICAL detector at INO is described in Section III. In Section IV a brief account of the atmospheric neutrino flux that has been used in the present analysis has been furnished. The generation of simulated data at ICAL and the analysis of such data are described in Section V. In Section VI we present the results and precision study of the oscillation parameters. Finally, Section VII includes discussions and conclusions.

\section{NEUTRINO OSCILLATION}

A neutrino flavor eigenstate $\left|v_{\alpha}\right\rangle(\alpha \equiv e, \mu, \tau$ etc. $)$ can be written as a linear superposition of neutrino eigenstates $\left|v_{i}\right\rangle$ (with definite non-degenerate masses $m_{i}$ ) in the mass basis as $\left|v_{\alpha}\right\rangle=\sum_{i} U_{\alpha i}\left|v_{i}\right\rangle\left(i=1,2,3\right.$ etc.). Here $U_{\alpha i}$ are the matrix elements of the neutrino mixing matrix $U$. This gives rise to the phenomenon of neutrino flavor oscillation. The probability that a neutrino $v_{g}$ with energy $E$ gets converted into another neutrino $v_{f}$ after traversing a distance $L$ in vacuum is given by

$$
\begin{aligned}
P\left(v_{g} \rightarrow v_{f}\right)=\delta_{f g} & -4 \sum_{j>i} \operatorname{Re}\left(U_{f i}^{*} U_{g i} U_{f j} U_{g j}^{*}\right) \sin ^{2}\left(1.27 \Delta m_{i j}^{2} \frac{L}{E}\right) \\
& \pm 2 \sum_{j>i} \operatorname{Im}\left(U_{f i}^{*} U_{g i} U_{f j} U_{g j}^{*}\right) \sin \left(2.54 \Delta m_{i j}^{2} \frac{L}{E}\right)(1)
\end{aligned}
$$

In the above, $L$ is expressed in $\mathrm{km}, E$ in $\mathrm{GeV}$ and $\Delta m^{2}$ in $\mathrm{eV}^{2}$. The $-(+)$ refers to neutrinos (anti-neutrinos).

For a two flavor scenario the above equation takes a simplified form given by

$$
P_{\text {survival }}=1-\sin ^{2} 2 \theta \sin ^{2}\left(1.27 \Delta m^{2} \frac{L}{E}\right)
$$

where $\theta$ is the mixing angle of neutrinos. Herein and in the rest of the paper the symbol $\theta$ and $\Delta m^{2}$ refer to $\theta_{23}$ and $\Delta m_{32}^{2}$.

\section{THE INO DETECTOR}

The simulation has been carried out for a detector with 50 $\mathrm{kTon}$ mass with dimension $48 \mathrm{~m} \times 16 \mathrm{~m} \times 12 \mathrm{~m}$ for ICAL [25]. The detector consists of a stack of 140 horizontal layers of $6 \mathrm{~cm}$ thick iron slabs interleaved with $2.5 \mathrm{~cm}$ gap for the active detector elements. For the sake of illustration, we define a rectangular coordinate frame with origin at the center of the detector, $x(y)$-axis along the longest (shortest) lateral direction, and $z$-axis along the vertical direction. A magnetic field of strength 1 Tesla is considered along the $+y$-direction. Resistive plate chambers (RPC) have been chosen as the active part of the detector. The readout of the RPCs is through the $\mathrm{Cu}$ strips having $2 \mathrm{~cm}$ width and placed orthogonally on the two external sides of the detectors. This type of detector has good time ( $\sim 1 \mathrm{~ns})$ and spatial resolutions.

\section{ATMOSPHERIC NEUTRINO FLUX}

The atmospheric neutrinos are produced from the interactions of the cosmic rays with earth's atmosphere. The knowledge of the primary spectrum of cosmic rays has been improved from the observations by BESS [32, 33] and AMS [34]. However, a large region of parameter space has been unexplored and they are interpolated or extrapolated from the measured flux. The difficulties and uncertainties in the calculation of the neutrino flux depend on the neutrino energy [35]. The low energy flux is known quite well. The cosmic ray fluxes $(<10 \mathrm{GeV})$ are modulated by solar activity and the geomagnetic field through a rigidity (momentum/charge) cutoff. At higher neutrino energy $(>100 \mathrm{GeV})$, solar activity and rigidity cutoff are irrelevant. There is an agreement within 5\% among the calculations for neutrino energy below $10 \mathrm{GeV}$ though different groups used different hadronic interaction models in their calculations.

We use the neutrino interaction cross section model of NUANCE [36] incorporating a typical Honda flux calculated in a (1) 3-dimensional scheme [35].

\section{DATA GENERATION AND METHOD OF ANALYSIS}

The interactions of neutrinos with the detector material are simulated by the Monte Carlo method in NUANCE [36]. In order to study the ICAL detector response for each event we use another Monte Carlo code GEANT [37]. The GEANT code uses the information of vertex position and momentum of the product particles obtained from the output of NUANCE 
simulation.

Event reconstruction: Our present analysis is based on the tracks generated by the muons that are produced by the charged current (CC) interactions of the neutrinos in the detector volume. The muons lose energy mainly due to ionization and radiative processes. This energy loss is proportional to the effective path-length which is the product of geometric path-length and the density of the medium. This can be applied only for fully contained (FC) events.

In this simulation study we do not consider any atmospheric muon background and the noise hits produced by the detector. However, we conservatively assumed hits $>6$ will be required for the reconstruction and filtering of the muon events from the latter background.

For a given triggered energy of a muon, the number of hits decreases when one goes from vertical to horizontal direction since it traverses less number of active detector elements. This dependence on the direction is less for the effective pathlength.

In case of partially contained (PC) events the momentum has been determined from the curvature of the track at the vertex due to the magnetic field applied across the detector. Due to limitation of our PC event reconstruction algorithm we considered PC events with hits $<20$.

On the other hand, the hadrons produced create a shower of hits around the vertex of the event. This implies that for any particular CC event the longest track normally comes from muons and this can be utilized for the analysis.

The up-going muon type neutrinos traverse larger pathlength undergoing oscillation whereas the down-going ones with much shorter path-length have little chance to oscillate. So one can visualize our detector set up as far (near) detector for the up-going (down-going) neutrinos. Then the ratio of up-going and down-going neutrinos (up/down) will roughly mimic the survival probability. This up/down ratio as a function of $L / E$ minimizes the systematic uncertainties in flux as well as in cross sections. The length $L$ for the up-going neutrinos is the actual path-length traversed by them whereas for down-going neutrinos the reference path-length $L$ is considered to be that of associated up-going neutrinos with zenith angle $\left(180^{\circ}-\theta_{\text {zenith }}\right)$ so that the range of $L / E$ remains the same for up-going and down-going neutrinos [38].

\section{Selection of events and Resolutions:}

The $L / E$ resolution has a complicated dependence on $L$ and $E$. However, a few general remarks can be made here. Qualitatively for a fixed energy, the $L$ resolution worsens gradually as we go from vertical to horizontal region and worsens

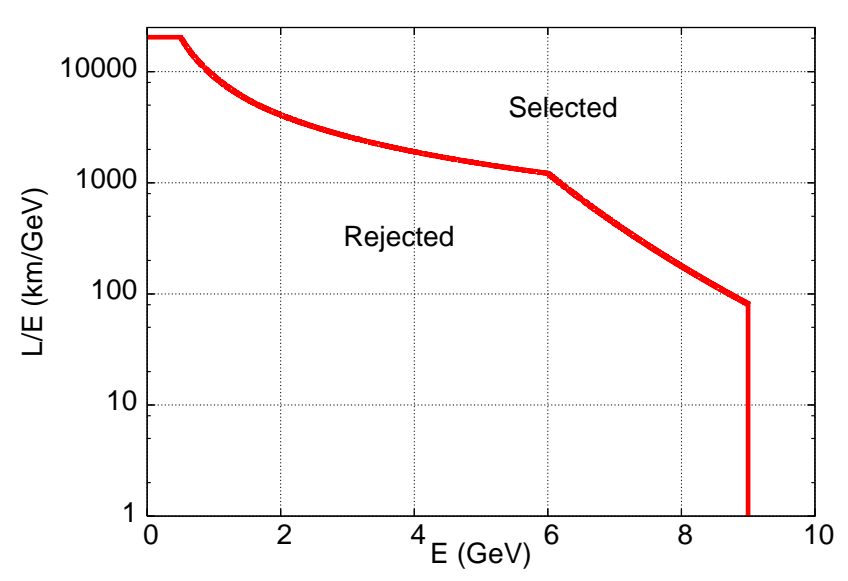

FIG. 1: The selection of events in $E-L / E$ plane, which gives good optimization between statistics and $L / E$ resolution.

rapidly close to the horizon. Also for a fixed direction, the $L / E$ resolution improves with increase in $E$. If one neglects totally the near horizon events (say, between zenith angle $70^{\circ}$ and $110^{\circ}$ ) all the events below $200 \mathrm{~km} / \mathrm{GeV}$ are lost. In our analysis we consider only the high energy events at near horizon and relax it gradually as we move away from the horizon. Quantitatively, this is taken care of by an $E$ dependent cut of the form :

$$
L / E \geq a E^{b}
$$

broken into three segments as shown in fig. 1

Using the above cut the resolutions for $E, L$ and $L / E$ obtained with the atmospheric neutrino flux for the whole range of $E_{v}$ and $L_{v}$ are shown in fig. 2]

A representative statistics for 5 year data is shown in table II. Here the number of events with hits $>6$ is considered for the analysis. The selected number of events is further reduced by imposing the above $E$ dependent $L / E$ cut for a better resolution.

\begin{tabular}{|c|c|c|c|c|}
\hline \multirow{2}{*}{ cut } & \multicolumn{3}{|c|}{ No. of surviving events/efficiency } \\
\cline { 2 - 5 } & FC & efficiency & FC+PC & efficiency \\
\hline hit $>6$ & 4160 & - & 5351 & - \\
(for reconstruction) & & & & \\
\hline $\begin{array}{c}E \text { dependent } L / E \text { cut } \\
\text { (improves } L / E \text { resolution) }\end{array}$ & 2089 & $50.2 \%$ & 2808 & $52.4 \%$ \\
\hline
\end{tabular}

TABLE I: Sample number of events after cuts in 5-year data for $\Delta m^{2}=2.3 \times 10^{-3} \mathrm{eV}^{2}$.

Using the above cuts we find the up/down distribution for different time exposures of the ICAL detector. Here our main 

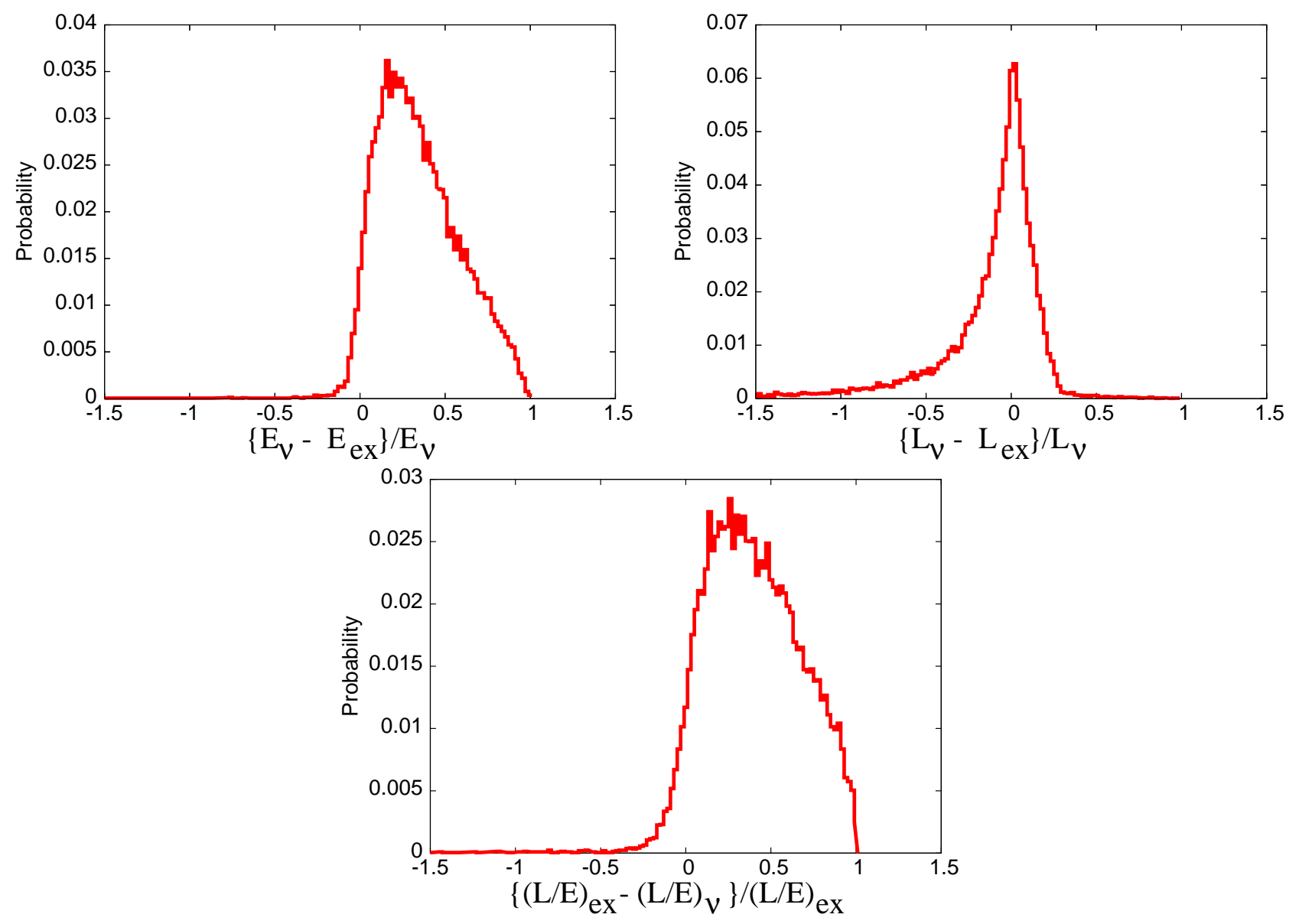

FIG. 2: The ICAL resolutions for $E, L$ and $L / E$ with atmospheric neutrinos for the whole range of $E_{\mathrm{v}}$ and $L_{v}$. The subscript 'ex' and $v$ refer to the reconstructed and true $v$ values.

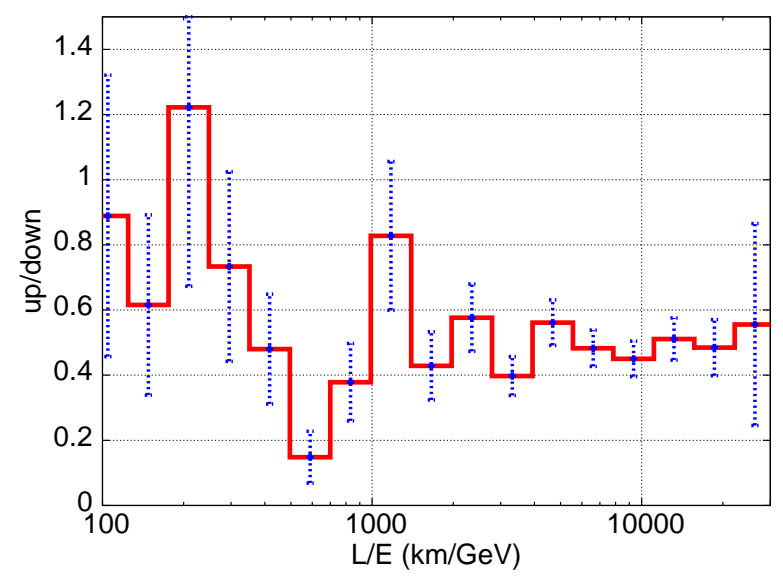

FIG. 3: The simulated up/down distribution at ICAL as a function of $L / E$ for 5 years FC events with $\Delta m^{2}=2.3 \times 10^{-3} \mathrm{eV}^{2}$.

goal is to find how precisely one can measure $\Delta m^{2}$. A representative 'up/down' distribution with respect to $L / E$ for 5 year FC events is shown in fig. 3. Such simulated plots are referred to as the 'experimental up/down' distributions.

$\chi^{2}$-analysis:
In the $\chi^{2}$-analysis the 'theoretical up/down' distribution is obtained by taking 40 years of atmospheric un-oscillated charged current muon neutrino data. The oscillation probability is then calculated from the $L$ and $E$ of the neutrinos for each event and the event is kept or rejected by throwing a ran- 
dom number. We smear this over the whole range of $L / E$ following the $L / E$-resolution function. Finally the up/down ratio is calculated for different $L / E$ bins. In this process we are also minimizing the effects due to geomagnetism and the shape of the earth.

Then a $\chi^{2}$-fit is made with the 'experimental up/down' distribution varying the atmospheric mass square difference $\Delta m^{2}$ and the mixing angle $\theta$ in the 'theoretical up/down' data.

\section{RESULTS}

The $L / E$ plot in fig. 3 shows clearly a full oscillation cycle and is typical for the ICAL detector. Thus ICAL is in a position to observe the oscillation pattern better than previous attempts, like the one by Super-K [8].

We show in fig. 4 the contours in the $\Delta m^{2}-\sin ^{2} 2 \theta$ plane for $90 \%$ and $99 \%$ CL with 5 year FC (upper left) and 5 year $\mathrm{FC}+\mathrm{PC}$ (upper right) events for the input value of $\Delta m^{2}=2.5 \times$ $10^{-3} \mathrm{eV}^{2}$ and with 10 years FC events (lower) for $\Delta m^{2}=2.7 \times$ $10^{-3} \mathrm{eV}^{2}$.

It is noted that the extracted best-fit values gradually become close to the input value of $\Delta m^{2}$ with increase of exposure time. For example, the best-fit value is found to be 2.50, 2.40 $(2.50,2.34) \times 10^{-3} \mathrm{eV}^{2}$ for 5,10 years $\mathrm{FC}(\mathrm{FC}+\mathrm{PC})$ events with the input $2.3 \times 10^{-3} \mathrm{eV}^{2}$. For all these cases the bestfit values of $\sin ^{2} 2 \theta$ turns out to be 1 with the input value 1 . Since the $\mathrm{FC}+\mathrm{PC}$ sample contains more high energy events than the FC sample and the $E$ and $L$ resolution are better for PC events, the best-fit values obtained from the FC+PC analysis are closer to the input value. With a change of the input $\Delta m^{2}$ from 2.5 to $2.7 \times 10^{-3} \mathrm{eV}^{2}$ for 10 year FC samples, the best-fit changes to 2.46 to $2.68 \times 10^{-3} \mathrm{eV}^{2}$.

The position of the dip in this up/down distribution is indicative of the best-fit value of $\Delta m^{2}$ while the overall statistics determines the size of the allowed parameter space. Particularly, the statistics in the larger (smaller) $L / E$ region from the dip determines the lower (upper) bound of $\Delta m^{2}$. For atmospheric neutrinos the statistics increases with increase of $L / E$ thus resulting in a better lower bound in the contour of $\Delta m^{2}$.

We have made a comparison of the present results and those obtained by the Super-K [8, 39] and the MINOS [10] experiments. They are shown in fig. 5. Here we plot contours at $90 \%$ and $99 \% \mathrm{CL}$ in $\Delta m^{2}-\sin ^{2} 2 \theta$ plane as obtained from our study of 5 years FC data and those obtained from previous Super-K (1489 days data) and the recent MINOS data. For the case of Super-K, two different analyses, one with respect to zenith angle [39] and the other with respect to $L / E$ [8] are given. One can see clearly that ICAL results are far more pre- cise than those of MINOS and substantially better than those from Super-K.

\section{A. Precision}

We define precision $(\mathrm{P})$ for a certain confidence level of a particular set of oscillation parameters as

$$
\mathrm{P}=2\left(\frac{\mathrm{UL}-\mathrm{LL}}{\mathrm{UL}+\mathrm{LL}}\right)
$$

where 'UL' and 'LL' are the upper and lower limit of the contour respectively at the specified confidence level.

FC analysis: The variation of precision of $\sin ^{2} \theta$ and $\Delta m^{2}$ with different years of exposure is shown separately in fig. 6 at $90 \%$ and $99 \%$ CL. It is seen that the precision falls very slowly beyond ten years and that can be a useful observation for the future experiment.

It is further observed that the precision gradually becomes worse when we increase the value of $\Delta m^{2}$ from $2.3 \times 10^{-3} \mathrm{eV}^{2}$. This is demonstrated in fig. 7 .

The reason behind this is the following. The position of the dip in the up/down distribution shifts towards larger values of $L / E$ with the decrease of the value of $\Delta m^{2}$. The flux increases rapidly with decrease of energy and the statistics becomes gradually high at larger $L / E$.

However, we comment that there is a competition between statistics and $L / E$ resolution. We see that at low value of $\Delta m^{2}$, say $2.1 \times 10^{-3} \mathrm{eV}^{2}$, the precision worsens compared to that $2.3 \times 10^{-3} \mathrm{eV}^{2}$. This can be improved if we choose more stringent cuts with good $L / E$ resolution for larger values of $L / E$. So one has to optimize between the requirement of statistics and $L / E$-resolution, which depends mainly on the range of interest of $\Delta m^{2}$.

FC+PC analysis: After the inclusion of the PC events the results are very similar to that obtained from $\mathrm{FC}$ events and hence are not shown separately.

\section{DISCUSSIONS}

Simulation studies for atmospheric neutrinos at the proposed Iron Calorimeter (ICAL) detector at INO have been made with a goal to determine the level of precision which may be achieved. The oscillated atmospheric neutrino events for a known set of values of oscillation parameters are generated with the event generator code (NUANCE) and the simulated signals in the detector are obtained through the detector simulation code (GEANT) that uses the NUANCE output as 

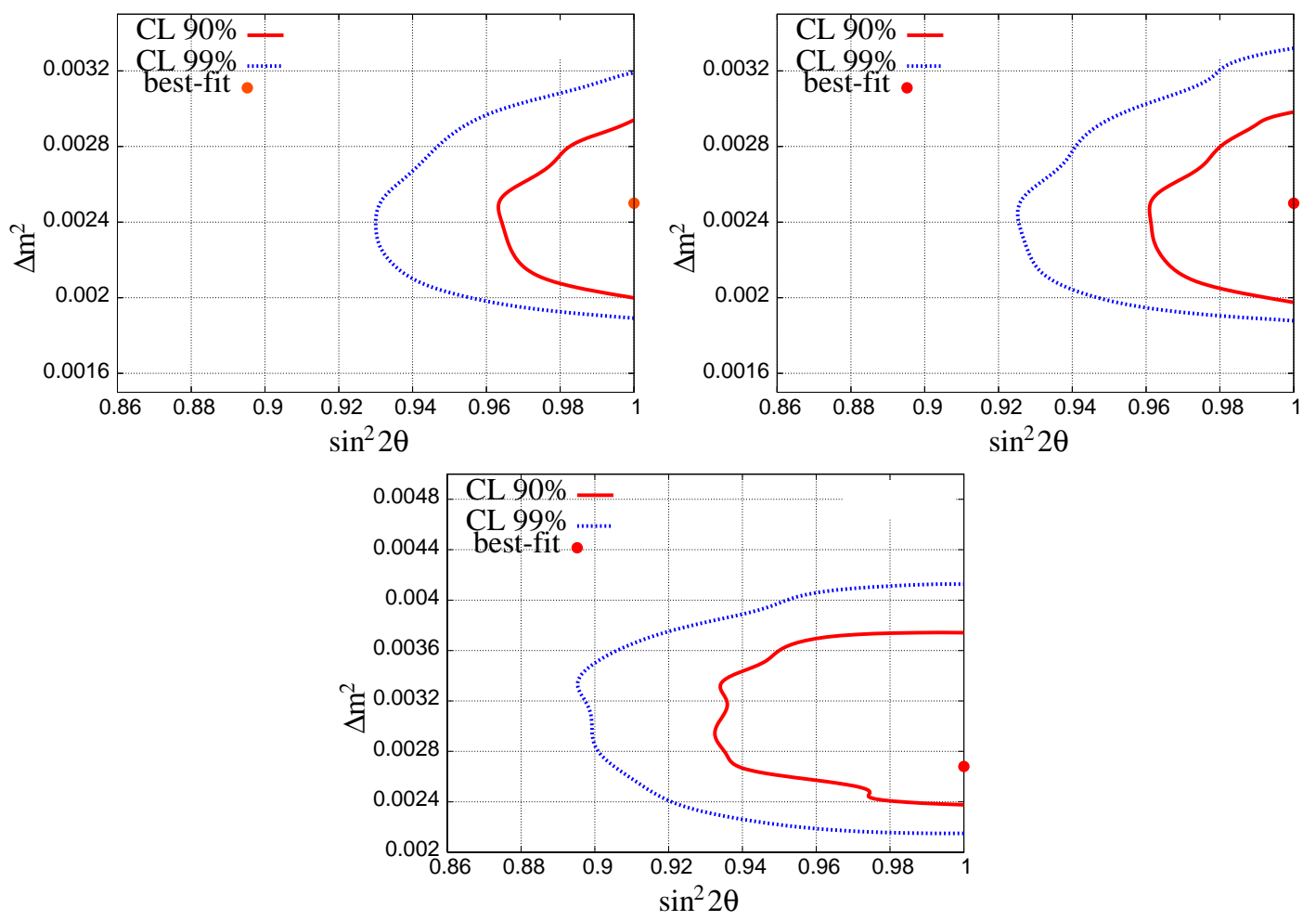

FIG. 4: The contour plots in $\Delta m^{2}-\sin ^{2} 2 \theta$ plane with input $\Delta m^{2}=2.3 \times 10^{-3} \mathrm{eV}^{2}$ for 5 years FC events (upper left), FC+PC event (upper right); the lower plot is with input $\Delta m^{2}=2.7 \times 10^{-3} \mathrm{eV}^{2}$ for 10 years $\mathrm{FC}$ events.

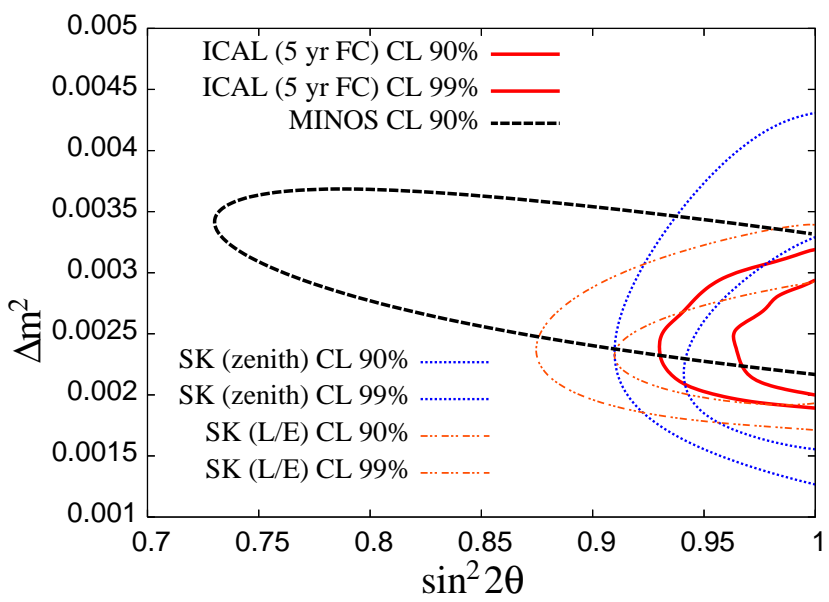

FIG. 5: The contours at $90 \%$ and $99 \% \mathrm{CL}$ for 5 years FC events with $\Delta m^{2}=2.3 \times 10^{-3} \mathrm{eV}^{2}$ at ICAL and the contours from the current experiments.

its inputs. A $\chi^{2}$ analysis of the results obtained from this simulated GEANT output data, properly chosen using appropriate constraints ("cuts"), is performed for the precision studies.

There is, however, scope for improvement of these studies.

- The present analysis is performed only with the simulated muon signals neglecting the hadrons. The estimation of neutrino energy $E$ and $L / E$ is expected to improve by the inclusion of hadrons. As hadrons mainly produce showers instead of well defined tracks, the method of using tracklength is not effective to extract energy information (or directional information) from such hadron showers. A new methodology is to be developed for this purpose and this work of incorporating hadrons in the analysis is in progress.

- A full three flavor analysis can address issues like mat- 

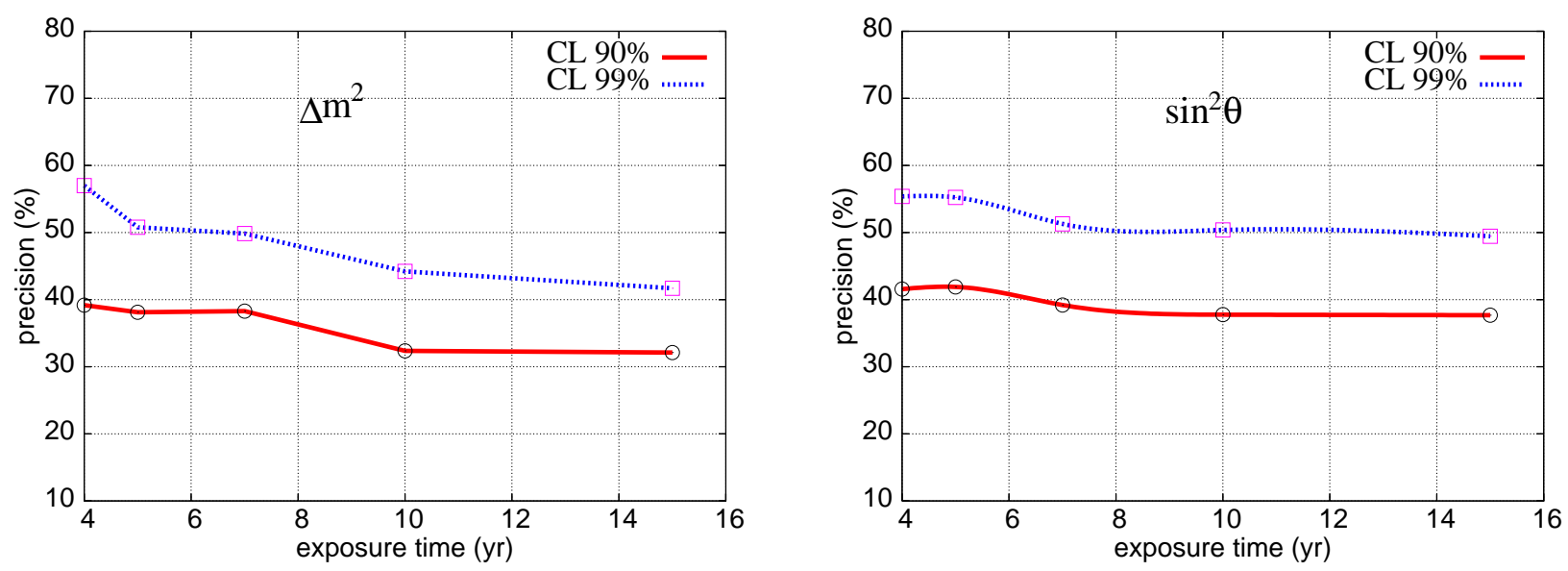

FIG. 6: The variation of the precision of $\Delta m^{2}$ (left) and $\sin ^{2} \theta$ (right) with time of exposure for 50kTon ICAL with FC events for the input of $\Delta m^{2}=2.3 \times 10^{-3} \mathrm{eV}^{2}$.
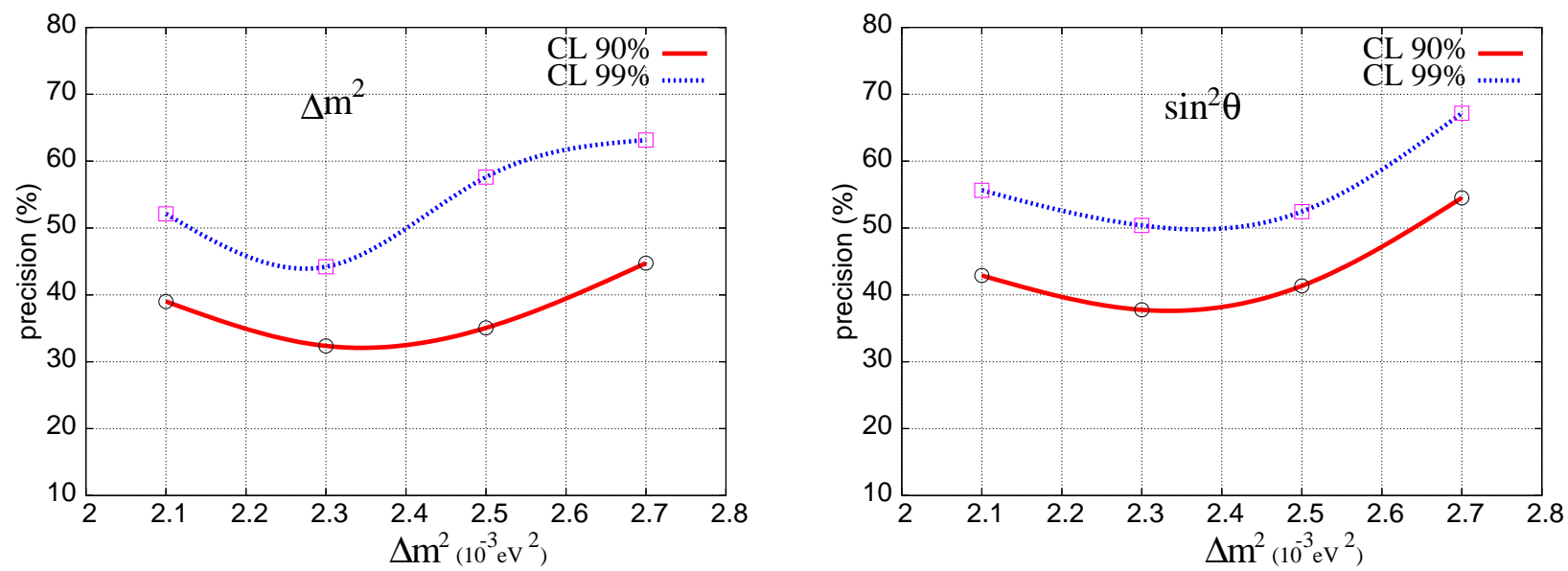

FIG. 7: The variation of the precision of $\Delta m^{2}$ (left) and $\sin ^{2} \theta$ (right) with the input value of $\Delta m^{2}$ for 50kTon ICAL with 10 years FC events.

ter effect, mass hierarchy and the deviation from maximality of the atmospheric mixing.

- For the analysis of PC events the curvature of the track is used for calculation of energy. In the high energy regime the tracks have no or negligible curvature inside the detector volume and hence such PC events could not be considered in the present analysis.

Moreover, we find the resolutions are energy dependent and significantly different for neutrinos and anti-neutrinos. One therefore expects to obtain more precise best fit values with
CL contours in parameter space further shrunk, if one uses multiple resolution functions, instead of one as used here. In doing so, the whole $L-E$ plane is divided into multiple small segments (mesh) and separate resolution functions are obtained for each such segment of the mesh which is then used for the purpose of analysis.

\section{Acknowledgements}

The authors thank Gobinda Majumder, Subhendu Rakshit, Sunanda Banerjee, Subhashis Chattopadhyay and Naba K Mondal for their help and valuable suggestions at different phases of the work.
[1] Y. Fukuda et al. [Super-Kamiokande Collaboration], Phys. Rev. Lett. 81, 1562 (1998) [arXiv:hep-ex/9807003].
[2] S. Eidelman et al. [Particle Data Group], Phys. Lett. B 592, 1 (2004). 
[3] B. Pontecorvo, Sov. Phys. JETP 6, 429 (1957).

[4] Z. Maki, M. Nakagawa and S. Sakata, Prog. Theor. Phys. 28, 870 (1962).

[5] T. Schwetz, Phys. Scripta T127, 1 (2006) [arXiv:hep-ph/0606060].

[6] M. Apollonio et al. [CHOOZ Collaboration], Phys. Lett. B 466, 415 (1999) arXiv:hep-ex/9907037.

[7] A. Bandyopadhyay, S. Choubey, S. Goswami, S. T. Petcov and D. P. Roy, Phys. Lett. B 608, 115 (2005) arXiv:hep-ph/0406328].

[8] Y. Ashie et al. [Super-Kamiokande Collaboration], Phys. Rev. Lett. 93, 101801 (2004) [arXiv:hep-ex/0404034].

[9] M. G. Zois, FERMILAB-MASTERS-2004-06.

[10] D. G. Michael et al. [MINOS Collaboration], Phys. Rev. Lett. 97, 191801 (2006) [arXiv:hep-ex/0607088].

[11] Y. Yamada [T2K Collaboration], Nucl. Phys. Proc. Suppl. 155, 207 (2006).

[12] J. Kisiel [ICARUS Collaboration], Acta Phys. Polon. B 36, 3227 (2005).

[13] A. Rubbia [ICARUS-CERN-Milano Collaboration], Nucl. Phys. Proc. Suppl. 66, 436 (1998).

[14] R. Ray, Nucl. Phys. Proc. Suppl. 154, 179 (2006).

[15] D. A. Harris [MINOS and NOvA Collaborations], Nucl. Phys. Proc. Suppl. 149, 150 (2005).

[16] G. Horton-Smith [Double Chooz Collaboration], AIP Conf. Proc. 805, 142 (2006).

[17] D. Motta [Double Chooz Collaboration], Acta Phys. Polon. B 37, 2027 (2006).

[18] C. K. Jung, arXiv:hep-ex/0005046

[19] H. Back et al., arXiv:hep-ex/0412016

[20] Y. Itow et al., arXiv:hep-ex/0106019

[21] K. Nakamura, Int. J. Mod. Phys. A 18, 4053 (2003).

[22] A. G. Cocco [OPERA Collaboration], Nucl. Phys. Proc. Suppl. 85, 125 (2000).

[23] C. Gustavino [OPERA Collaboration], J. Phys. Conf. Ser. 39,
326 (2006).

[24] F. Di Capua [OPERA Collaboration], PoS HEP2005, 177 (2006).

[25] M. S. Athar et al. [INO Collaboration], INO-2006-01.

[26] D. Indumathi and M. V. N. Murthy, Phys. Rev. D 71, 013001 (2005) arXiv:hep-ph/0407336.

[27] R. Gandhi, P. Ghoshal, S. Goswami, P. Mehta and S. Uma Sankar, Phys. Rev. D 73053001 (2006) arXiv:hep-ph/0411252.

[28] S. T. Petcov and T. Schwetz, Nucl. Phys. B 740, 1 (2006) arXiv:hep-ph/0511277.

[29] A. Samanta, arXiv:hep-ph/0610196.

[30] S. Choubey and P. Roy, Phys. Rev. D 73, 013006 (2006) |arXiv:hep-ph/0509197|.

[31] D. Indumathi, M. V. N. Murthy, G. Rajasekaran and N. Sinha, Phys. Rev. D 74, 053004 (2006) arXiv:hep-ph/0603264.

[32] T. Sanuki et al., Astrophys. J. 545, 1135 (2000) arXiv:astro-ph/0002481.

[33] T. Maeno et al. [BESS Collaboration], Astropart. Phys. 16, 121 (2001) arXiv:astro-ph/0010381.

[34] J. Alcaraz et al. [AMS Collaboration], Phys. Lett. B 490, 27 (2000).

[35] M. Honda, T. Kajita, K. Kasahara and S. Midorikawa, Phys. Rev. D 70, 043008 (2004) [arXiv:astro-ph/0404457].

[36] D. Casper, Nucl. Phys. Proc. Suppl. 112, 161 (2002) |arXiv:hep-ph/0208030|.

[37] GEANT Detector Simulation and Simulation Tool, CERN Program Library Long Write-up W5013, March 1994, http://wwwasd.web.cern.ch/wwwasd/cernlib/version.html

[38] P. Picchi and F. Pietropaolo, ICGF RAP. INT. 344/1997, Torino 1997 (CERN preprint SCAN-9710037).

[39] Y. Ashie et al. [Super-Kamiokande Collaboration], Phys. Rev. D 71, 112005 (2005) [arXiv:hep-ex/0501064]. 